ORIGINAL ARTICLE

\title{
CD133 positive endothelial progenitor cells contribute to the tumour vasculature in non-small cell lung cancer
}

\author{
W Hilbe, S Dirnhofer, F Oberwasserlechner, T Schmid, E Gunsilius, G Hilbe, E Wöll, C M Kähler
}

J Clin Pathol 2004;57:965-969. doi: 10.1136/icp.2004.016444

See end of article for authors' affiliations

.....................

Correspondence to:

Dr W Hilbe, Department of Internal Medicine,

Innsbruck University

Hospital, Anichstrasse 35,

A-6020 Innsbruck,

Austria; wolfgang.hilbe@

vibk.ac.at

Accepted for publication 24 March 2004
Aims: Recent results generated in a mouse model suggest that tumour angiogenesis/vasculogenesis can be initiated and maintained by bone marrow derived endothelial progenitor cells. This present study investigated the distribution and frequency of CD133 positive endothelial progenitor cells in patients with non-small cell lung cancer (NSCLC) (tumour tissue and tumour free lung regions) and healthy controls using fresh frozen specimens. The novel marker CD133 identifies human haemopoetic precursor cells, in addition to human endothelial progenitor cells.

Methods: Seventy nine lung cancer specimens and 66 adjacent histologically tumour free tissues of the same patient cohort were analysed; 11 postmortem specimens from control patients who did not suffer from malignant disease served as controls. Cryostat sections were stained for CD133, CD31, vascular endothelial growth factor receptor 2 (VEGFR-2; KDR), p53, and the proliferation marker Ki-67, and the correlations were analysed.

Results: Forty three of 63 evaluable tumour specimens had increased numbers of CD133 positive cells and in some cases capillary forming CD133 positive structures were detectable. In addition, 30 of 63 specimens had raised expression of KDR and 29 of 63 had increased MVD. Increased CD133 expression marginally correlated with raised KDR expression but not with p53 and Ki-67.

Conclusion: A significant increase in CD133 positive cells was documented in patients with NSCLC, suggesting an involvement of endothelial progenitor cells in tumour vasculogenesis and tumour growth in these patients.
A ngiogenesis is essential for tumour growth and metastatic spread and represents an important prognostic indicator in non-small cell lung cancer (NSCLC). ${ }^{1}$ Because different anti-angiogenic substances are currently under development and some of them have already been tested in clinical trials, the determination of the neoangiogenic potential of malignant tumours is of pivotal interest. $^{2-5}$

The growth of mature endothelial cells (ECs) depends on different growth factors leading to the recruitment of ECs from neighbouring pre-existing capillaries. ${ }^{6-8}$ Vascular endothelial growth factor (VEGF) is one of the key stimuli of endothelial proliferation and migration, and thus plays an essential role in physiological and pathological angiogenesis, and has also been reported to correlate with the neoangiogenesis occurring during cancer. ${ }^{9}$ Furthermore, VEGF is one of the key molecules in recruiting and activating endothelial progenitor cells (EPCs) from the bone marrow. ${ }^{10}$ In humans, increased immunolocalisation of VEGF in NSCLC specimens seems to be associated with a poor outcome. ${ }^{11-13}$ However, the impact of increased VEGF expression alone on tumour angiogenesis is ambiguous, ${ }^{10}{ }^{12} 14$ and the determination of VEGF alone seems to be an inadequate means of defining angiogenic activity in NSCLC.

Furthermore, recent evidence suggests that the recruitment of ECs from neighbouring pre-existing capillaries is not the only source of increased tumour vascularisation during cancer spread. Lyden and colleagues ${ }^{15}$ demonstrated in a mouse model that the recruitment of bone marrow derived endothelial precursors plays an essential role in tumour vascularisation and tumour growth. These bone marrow derived endothelial cells are thought to contribute to angiogenic processes in cancer tissue and also to have the capacity to form new vessels-a process known as vasculogenesis. EPCs represent an immature subset of CD34 positive cells expressing the $120 \mathrm{kDa}$ glycosylated polypeptide CD133. ${ }^{16} 17$ In contrast, mature ECs present in pre-existing vessels are CD133 negative. ${ }^{16}$ Interestingly, a more recent study reported that increased numbers of circulating CD133 positive EPCs occur in patients with breast cancer. These findings support the hypothesis of a functional role of EPCs in vascularisation and growth of human solid organ tumours. $^{18}$

"Endothelial progenitor cells represent an immature subset of CD34 positive cells expressing the 120 kDa glycosylated polypeptide CD133"

Until now, the standard method of measuring tumour angiogenesis has been the determination of intratumorous microvessel density (MVD), which is closely correlated with tumour growth and postoperative prognosis. Thus, patients with highly vascularised tumours suffer from early metastatic disease and reduced survival. ${ }^{19-24}$

Multiple markers have been found to be altered in patients with NSCLC and some of them are thought to influence vascularisation of the tumour. Mutated p53 has been shown to potentiate the induction of VEGF secretion and to reduce the expression of the antiangiogenic factor thrombospondin $1 .{ }^{25} \mathrm{Bcl}-2$ and $\mathrm{p} 53$ have been shown to regulate VEGF mediated angiogenesis in NSCLC. ${ }^{27}$ In addition, the proliferation associated marker $\mathrm{Ki}-67^{28}$ is an essential prognostic parameter for NSCLC.

Abbreviations: EC, endothelial cell; EPC, endothelial progenitor cell; MVD, microvessel density; NSCLC, non-small cell lung cancer; VEGF, vascular endothelial growth factor; VEGFR, vascular endothelial growth factor receptor 
Here, we show for the first time that increased numbers of CD133 positive EPCs are found in NSCLC specimens and that they may contribute to tumour vasculogenesis. Furthermore, the relation between this increase and VEGF receptor 2 (VEGFR-2; KDR) expression and its correlation with MVD, proliferative activity, and p53 expression is presented.

\section{PATIENTS AND METHODS Patients}

Fresh tumour tissues from 79 patients with NSCLC were obtained for routine diagnosis. All patients were treated surgically with curative intention. One portion of the sample was fixed in 10\% formalin and another part was immediately snap frozen in liquid nitrogen and stored at $-80^{\circ} \mathrm{C}$ for immunohistology. All tumour specimens were evaluated histopathologically according to the World Health Organisation classification. ${ }^{29}$

As a result of an alveolar pattern of tumour infiltration, with consecutively increased numbers of capillaries, 16 patients were excluded from further evaluation, because in these cases the determination of MVD could have produced false positive results. In the remaining 63 patients, the histological diagnosis was as follows: 25 cases of adenocarcinoma, 34 cases of squamous cell carcinoma, two cases of large cell carcinoma, and two cases of carcinoid tumour. According to UICC recommendations, 42 patients were classified as stage I, 13 patients as stage II, and eight patients as stage III. Patients in advanced clinical stages were treated with chemotherapy and/or radiotherapy.

\section{Immunocytochemical staining}

Frozen specimens were cut at $3-5 \mu \mathrm{m}$ in a cryostat, fixed, incubated with monoclonal antibodies at appropriate dilutions, and then incubated with an alkaline phosphatase complex (D651; Dako, Glostrup, Denmark), as reported previously. ${ }^{30}$ All antibodies were diluted with phosphate buffered saline with $1 \%$ bovine serum albumin. The following monoclonal antibodies were used: CD133/1 (clone AC133; Miltenyi Biotec, Bergisch Gladbach, Germany); anti-VEGFR2 (clone KDR-2; Sigma-Aldrich, St Louis, Missouri, USA); CD31 (clone WM59; Serotec, Kiddington, UK); PAb 1801 to detect p53 (clone 1801; Pharmingen, San Diego, California, USA), and Ki-67 (Dakopatts, Glostrup, Denmark).

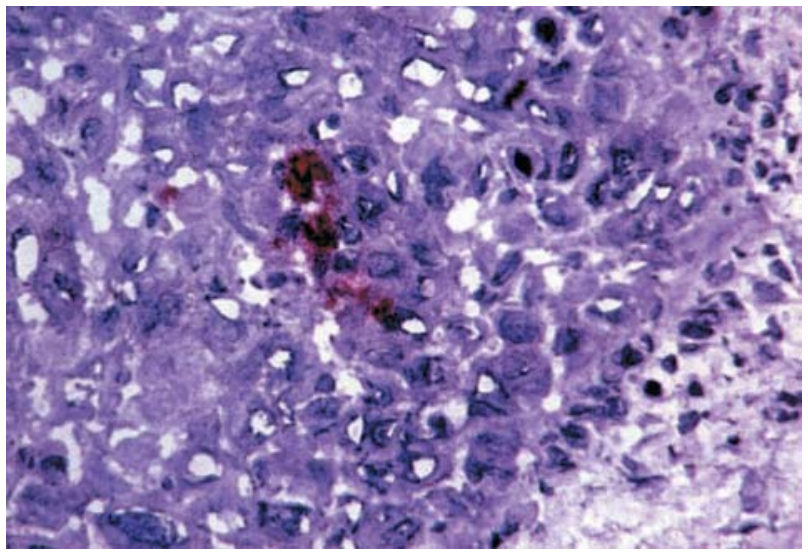

Figure 1 Immunostaining for $C D 133$ in a sequential section of a nonsmall cell lung cancer specimen; single CD133 positive cells are seen, together with initiation of endothelial progenitor cell mediated vasculogenesis. Alkaline phosphatase anti-alkaline phosphatase staining on cryostat section.

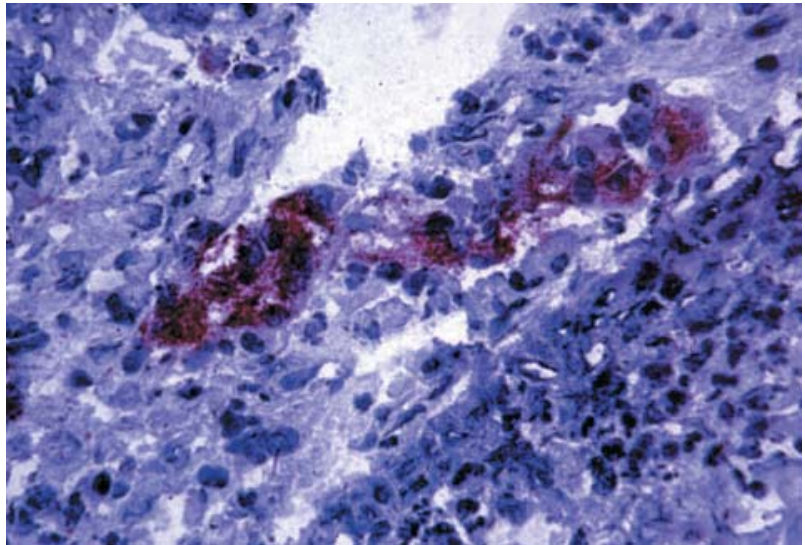

Figure 2 Immunostaining for $\mathrm{CD} 133$ in a sequential section of a nonsmall cell lung cancer specimen, showing a CD133 positive vessel. Alkaline phosphatase anti-alkaline phosphatase staining on cryostat section.

\section{Immunohistochemical evaluation}

Slides were evaluated independently by two of the authors (WH and SD) using a semiquantitative method on a Zeiss AXIOSKOP 2 microscope. Immunopositive cells were counted from representative areas of the sections and expressed as a percentage. The intensity of immunostaining was categorised as follows: - , negative; + , low; ++ , moderate; and +++ , high.

Cutoff values were defined by analysing adjacent histologically confirmed tumour free areas of 66 patients with NSCLC (mean percentage +2SD) ${ }^{30}$ : CD133, > 0.7\%; VEGFR-2 $(\mathrm{KDR}),>12.3 \% ; \mathrm{p} 53,>1.3 \%$; and $\mathrm{Ki}-67,>2.7 \%$. For VEGFR-2 expression a second cutoff was introduced, because the calculated value (12.3\%, "high expression") could not distinguish between the subgroups sufficiently. Therefore, the cutoff value was defined by using the median value of the tumour free cohort (2.5\%, "low expression"). For CD31, the cutoff was defined as the median number of positive cells of the three most intense areas: $15 \%$. Only cases with moderate $(++)$ or high $(+++)$ staining intensity and a percentage of immunopositive cells above the cutoff point were scored as "positive".

Microvessel counting was performed in accordance with previous reports, ${ }^{121}$ with slight modifications, using CD31. EPCs localised in capillary structures were characterised further by CD133 and VEGFR-2 (KDR). For all three tested antibodies, the most intense regions were identified under low magnification $(\times 100)$, after which the positive cells were

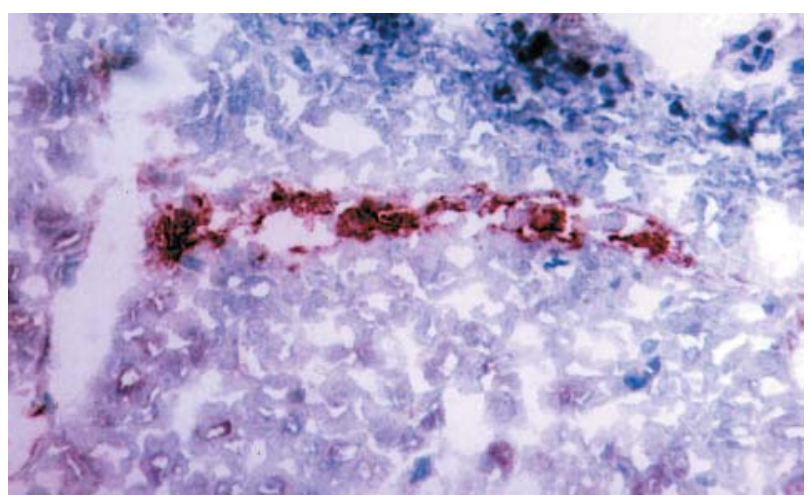

Figure 3 Immunostaining for CD31 in a sequential section of a nonsmall cell lung cancer specimen, showing a typical capillary structure embedded in the infiltrating tumour (CD31 positive). Alkaline phosphatase anti-alkaline phosphatase staining on cryostat section. 


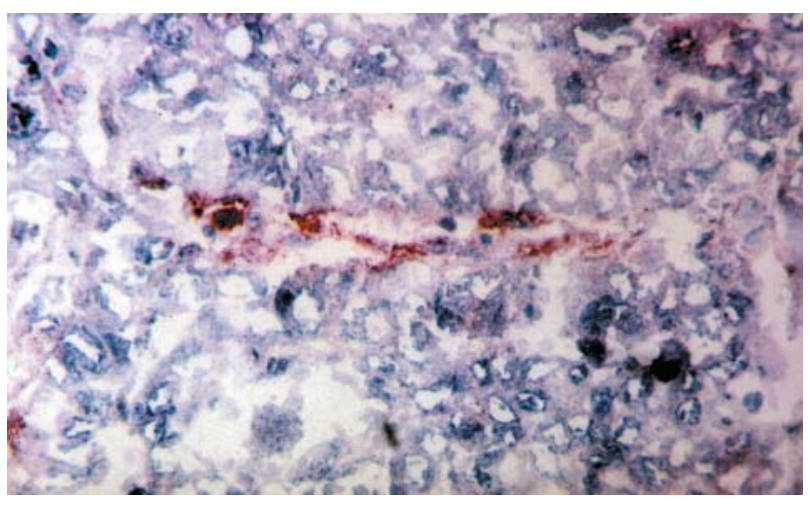

Figure 4 Immunostaining for CD133 in a sequential section of a nonsmall cell lung cancer specimen, same capillary as in fig 3 (CD133 positive). Alkaline phosphatase anti-alkaline phosphatase staining on cryostat section.

counted under higher magnification $(\times 400)$ in these regions. Ki-67 and p53 analysis was performed on the whole cell population.

\section{Summary of the procedure for immunohistochemical analysis}

(1) Counting the number of "immunopositive cells" expressed as a percentage.

(2) Assessing the intensity of immunostaining ( - , negative; + , low; ++ , moderate; and +++ , high).

(3) "Positive cases": moderate $(++)$ or high $(+++)$ staining intensity and a percentage of "immunopositive cells" above the cutoff points.

\section{Statistical analysis}

The Pearson $\chi^{2}$ test was used to compare the results of two or more subgroups. Significance was determined by means of 95\% confidence intervals. All statistical procedures were performed using SPSS, version 7.5, statistical software (SPSS Inc, Chicago, Illinois, USA)

\section{RESULTS}

\section{Patients}

The median age of the 63 patients evaluated was 62 (range, $37-79)$ years at the time of surgery. The male : female ratio was $4.2: 1$ ( $51: 12)$. The median observation time was 17 (range, 1-95) months, the median survival was not reached, and 18 patients died.

The analysis was based on three different groups of tissues, namely:
(1) Tumour specimens of patients with NSCLC (tumour group).

(2) Tumour free specimens of patients with NSCLC (tumour free group).

(3) Tumour free specimens of non-tumour patients (healthy control group).

\section{Immunohistochemistry}

Tables 1 and 2 give the results of the immunohistochemical staining. Increased numbers of CD133 positive cells were found in 43 of 63 cases within the tumour group, and some cases revealed capillary forming structures positive for CD133 staining (figs 1-4). Overall, the median number of CD133 positive cells within the tumour specimens was $0.2 \%$ (range, $0-50 \%)$ and 43 were over the cutoff value $(0.7 \%)$. In contrast, in tumour free samples $(n=66)$ of the same NSCLC patient cohort, only single CD133 positive cells were detected (median, 0\%; range, $0-1 \%$ ) and no $\mathrm{CD} 133$ positive capillary structures were seen. The same data were obtained in the control group (median, 0\%; range, 0-1\%). In the tumour group CD133 expression correlated marginally with VEGFR-2 expression (cutoff value of $2.5 \%$; $=0.053$ ).

Increased numbers of VEGFR-2 (KDR) immunopositive cells were detected in 30 of 63 samples within the infiltrating tumour using a cutoff value of $2.5 \%$. The median number of VEGFR-2 positive cells was $2 \%$ (range, $0-20 \%$ ). VEGFR-2 expression (median, 2.5\%; range, $0-20 \%$ ) in the tumour free regions of these patients revealed similar values: 32 of 66 scored positive. Applying the calculated cutoff value (12.3\%), five patients in the tumour group and four in the tumour free cohort had raised numbers of VEGFR-2 positive cells. Similar values were detected ( $2 \%$ VEGFR-2 positive cells, range, $0.2-$ $20 \%$; number of positive cases, four of 11 (cutoff, $2.5 \%$ ) or one of 11 (cutoff, 12.3\%)) in the control group. There were no significant differences between the three cohorts analysed. When a lower cutoff value was applied, the distribution of VEGFR-2 positive cases within the three groups still did not differ (cutoff, 2.5\%: 36\% control group $v 48 \%$ tumour free group $v 48 \%$ tumour group).

Increased MVD (as assessed by CD31 expression) was detected in 29 of the 63 tumour samples. MVD correlated with tumour grade $(\mathrm{p}=0.033)$. In detail, MVD positive cases revealed a higher rate of grade III tumours (15 of 29) when compared with MVD negative tumours (nine of 29).

The frequency of Ki-67 and p53 expression did not correlate with the angiogenic parameters (CD133, CD31/ MVD, and VEGFR-2 (KDR)). Within the analysed clinical parameters (sex, histology, and stage) the angiogenic markers were distributed equally in the tumour cohort (table 1).

AC, adenocarcinoma; MVD, microvessel density; SCC, squamous cell carcinoma; VEGFR-2 (KDR), vascular endothelial growth factor receptor 2 .

Table 1 Comparison of angiogenic markers with clinical parameters

\begin{tabular}{lllll}
\hline No $(\%)$ & MVD & VEGFR-2 & CD133 & All patients \\
\hline $\begin{array}{l}\text { No. of positive cases } \\
\text { Diagnosis }\end{array}$ & 29 & 30 & 43 & 63 \\
AC & $11(38 \%)$ & $11(37 \%)$ & $19(44 \%)$ & $25(40 \%)$ \\
SCC & $17(59 \%)$ & $17(57 \%)$ & $22(51 \%)$ & $34(54 \%)$ \\
Other & $1(3 \%)$ & $2(6 \%)$ & $2(4 \%)$ & $4(6 \%)$ \\
Stage & $17(59 \%)$ & $20(67 \%)$ & $30(70 \%)$ & $42(67 \%)$ \\
Stage 1 & $6(21 \%)$ & $4(13 \%)$ & $8(19 \%)$ & $13(21 \%)$ \\
Stage 2 & $6(21 \%)$ & $4(13 \%)$ & $5(11 \%)$ & $8(13 \%)$ \\
Stage 3 & $9(31 \%)$ & $9(40 \%)$ & $14(33 \%)$ & $18(29 \%)$ \\
Died & & &
\end{tabular}


Table 2 Expression profiles of the three angiogenic markers analysed

\begin{tabular}{|c|c|c|c|c|c|c|}
\hline \multirow{2}{*}{ Number } & \multicolumn{2}{|c|}{ Control group } & \multicolumn{2}{|c|}{$\begin{array}{l}\text { Non-tumour specimens from } \\
\text { NSCLC group }\end{array}$} & \multicolumn{2}{|c|}{ NSCLC specimens } \\
\hline & \multicolumn{2}{|l|}{11} & \multicolumn{2}{|l|}{66} & \multicolumn{2}{|l|}{63} \\
\hline \multicolumn{7}{|l|}{ CD133 } \\
\hline Number of positive cells (median, range) & $0 \%$ & $0-1 \%$ & $0 \%$ & $0-1 \%$ & $0.2 \%$ & $0-50 \%$ \\
\hline Cases above cutoff value $(0.7 \%$ ) (number, \%) & 0 & $0 \%$ & 0 & $0 \%$ & 43 & $68 \%$ \\
\hline \multicolumn{7}{|l|}{ VEGFR-2 } \\
\hline Number of positive cells (median, range) & $2 \%$ & $0.2-20 \%$ & $2.50 \%$ & $0-20 \%$ & $2 \%$ & $0-20 \%$ \\
\hline Low expression: cutoff $2.5 \%$ (number, $\%$ ) & 4 & $36 \%$ & 32 & $48 \%$ & 30 & $48 \%$ \\
\hline High expression: cutoff $12.3 \%$ (number, \%) & 1 & $9 \%$ & 4 & $6 \%$ & 5 & $8 \%$ \\
\hline \multicolumn{7}{|l|}{ MVD (CD31) } \\
\hline Number of positive cells (median, range) & NE & & NE & & $6 \%$ & $0-32 \%$ \\
\hline Cases above cutoff value (15) (number, \%) & NE & & NE & & 29 & $46 \%$ \\
\hline p53 (number, \%) & 0 & $0 \%$ & 1 & $2 \%$ & 25 & $63 \%$ \\
\hline Ki-67 (number, \%) & 0 & $0 \%$ & 1 & $2 \%$ & 32 & $41 \%$ \\
\hline
\end{tabular}

Comparison of the three cohorts: tumour free control group ("control group"), tumour free specimens from patients with NSCLC, and tumour specimens from patients with NSCLC.

MVD, microvessel density; NE, not evaluable; NSCLC, non-small cell lung cancer.

\section{Survival analysis}

None of the tested angiogenetic markers was significantly related to survival in univariate analysis in the tumour cohort.

\section{DISCUSSION}

Tumour vascularisation seems to be essential for tumour growth and metastatic spread. ${ }^{7}$ In addition to angiogenesisthe migration and sprouting of ECs from neighbouring pre-existing capillaries-tumour induced vasculogenesis mediated by bone marrow derived EPCs is thought to be a possible mechanism of vascularisation. ${ }^{15-17} 31-33$

Asahara et al showed that EPCs isolated from peripheral blood are incorporated into sites of active angiogenesis, and that EPCs might help augment collateral vessel growth within ischaemic tissue. ${ }^{31}$ Furthermore, EPCs are thought to contribute to pathological neovascularisation ${ }^{32}{ }^{33}$-for example, in sites of myocardial infarction, and Lyden et al demonstrated in a mouse model that EPCs seem to be key cells for tumour vascularisation and growth. ${ }^{15}$ Until recently, no data were available on the presence of EPCs in solid organ cancer, and their possible contribution to tumour induced vasculogenesis in humans. However, a recent study has shown that increased numbers of circulating CD133 positive EPCs can be found in patients with breast cancer. ${ }^{18}$

Here, we show for the first time that increased numbers of CD133 positive EPCs are present in patients with NSCLC, and that they seem to contribute to capillary forming units in invading tumour formations (figs 1-4). Increased numbers of CD133 expressing cells were found in 43 of the 63 samples, correlating marginally with KDR expression. Previous phenotypic analyses revealed that most CD133 positive cells derived from CD34 positive cells display endothelial features, including the expression of VEGFR-2 (KDR). ${ }^{17}{ }^{34}$ Not surprisingly, we found a correlation between CD133 and VEGFR-2 (KDR) expression in our present study. However, CD133 did not correlate with MVD, as defined by CD31 positivity. This result is not surprising because CD31 is expressed on activated endothelial cells and on non-activated ones. CD31 expression can also be detected in many other cell types present in cancer tissues. ${ }^{35}$

All three evaluated cohorts (NSCLC specimens, and tumour free tissue from patients and the control group) revealed similar levels of VEGFR-2 expression. A possible explanation for this observation might be that VEGFR-2 staining is not endothelial cell specific. VEGFR-2 is expressed on endothelial cells, alveolar type II cells, and on endothelial progenitor cells. ${ }^{36}$ In addition, normal lung tissue has a high

\section{Take home messages}

- Forty three of 63 patients with non-small cell lung cancer had increased expression of CD133 positive cells in their tumour tissue

- Thirty of 63 specimens also had raised expression of KDR and 29 of 63 had increased microvessel density

- These results suggest that endothelial progenitor cells (EPCs) are involved in tumour vasculogenesis and tumour growth in these patients

- Treatment strategies targeting the mobilisation of EPCs from the bone marrow and the homing of these cells into malignant tumours might be an alternative approach for the treatment of patients with cancer

density of blood vessels, and consequently has high VEGFR-2 expression. Thus, VEGFR-2 expression seems to be insufficient to discriminate highly active tumours.

"We have shown for the first time that increased numbers of CD133 positive endothelial progenitor cells can be found in non-small cell lung cancer tissue and that these cells seem to contribute to the formation of capillaries"

In conclusion, we have shown for the first time that increased numbers of CD133 positive EPCs can be found in NSCLC tissue and that these cells seem to contribute to the formation of capillaries. Consequently, our data suggest that CD133 positive EPCs contribute to vasculogenesis in solid tumours, and might regulate tumour growth in humans. Thus, treatment strategies targeting the mobilisation of EPCs from the bone marrow and the homing of these cells into malignant tumours might be an alternative approach for the treatment of patients with cancer.

\section{ACKNOWLEDGEMENTS}

This study was supported by the Association for Cancer Research, Innsbruck, Austria (Verein für Tumorforschung - Innsbruck).

\section{Authors' affiliations}

W Hilbe, F Oberwasserlechner, E Gunsilius, E Wöll, C M Kähler,

Department of Internal Medicine, University Hospital, Anichstrasse 35, A-6020 Innsbruck, Austria 
S Dirnhofer, Institute of Pathology, University of Basel, CH 4003 Basel, Switzerland

T Schmid, Department of Surgery, University Hospital, Innsbruck

G Hilbe, Department of Surgery, Natters State Hospital, Austria

\section{REFERENCES}

1 Fontanini G, Lucchi M, Vignati S, et al. Angiogenesis as a prognostic indicator of survival in non-small-cell lung carcinoma: a prospective study. J Natl Cancer Inst 1997;89:881-6.

2 Schnitzer JE. Vascular targeting as a strategy for cancer therapy. NEngl J Med 1998;339:472-4.

3 Dvorak HF. Vascular permeability factor/vascular endothelial growth factor: a critical cytokine in tumor angiogenesis and a potential for diagnosis and therapy. J Clin Oncol 2002;20:4368-80.

4 Klement G, Baruchel S, Rak J, et al. Continuous low-dose therapy with vinblastine and VEGF receptor-2 antibody induces sustained tumor regression without overt toxicity. J Clin Invest 2000;105:R15-24.

5 Gordon MS, Margolin K, Talpaz M, et al. Phase I safety and pharmacokinetic study of recombinant human anti-vascular endothelial growth factor in patients with advanced cancer. J Clin Oncol 2001;19:843-50.

6 Soncin F, Vandenbunder B. Tumor angiogenesis. In: Brambilla C, Brambilla E, eds. Lung tumors: fundamental biology and clinical management. New York: Marcel Dekker, 1999:365-97.

7 Folkman J, Watson K, Ingber D, et al. Induction of angiogenesis during the transition from hyperplasia to neoplasia. Nature 1989;339:58-61.

8 Yancopoulos GD, Davis S, Gale NW, et al. Vascular-specific growth factors and blood vessel formation. Nature 2000;407:242-8.

9 Tsao MS, Liu N, Nicklee T, et al. Angiogenesis correlates with vascular endothelial growth factor expression but not with $\mathrm{Ki}$-ras oncogene activation in non-small cell lung carcinoma. Clin Cancer Res 1997;3:1807-14.

10 Asahara T, Takahashi T, Masuda H, et al. VEGF contributes to postnatal neovascularization by mobilizing bone marrow-derived endothelial progenitor cells. EMBO J 1999; 18:3964-72.

11 Koukourakis MI, Giatromanolaki A, Thorpe PE, et al. Vascular endothelial growth factor/KDR activated microvessel density versus CD31 standard microvessel density in non-small cell lung cancer. Cancer Res 2000;60:3088-95.

12 Giatromanolaki A, Koukourakis Ml, Kakolyris S, et al. Vascular endothelial growth factor, wild-type p53, and angiogenesis in early operable non-smal cell lung cancer. Clin Cancer Res 1998;4:3017-24.

13 Ohta Y, Endo Y, Tanaka M, et al. Significance of vascular endothelial growth factor messenger RNA expression in primary lung cancer. Clin Cancer Res 1996;2:1411-16.

14 Gunsilius E, Tschmelitsch J, Eberwein M, et al. In vivo release of vascular endothelial growth factor from colorectal carcinomas. Oncology 2002;62:313-17.

15 Lyden D, Hattori K, Dias S, et al. Impaired recruitment of bone-marrowderived endothelial and hematopoietic precursor cells blocks tumor angiogenesis and growth. Nat Med 2001;7:1194-201.

16 Yin AH, Miraglia S, Zanjani ED, et al. AC133, a novel marker for human hematopoietic stem and progenitor cells. Blood 1997;90:5002-12.
17 Gehling UM, Ergun S, Schumacher U, et al. In vitro differentiation of endothelial cells from AC133-positive progenitor cells. Blood 2000;95:3106-12.

18 Sussman LK, Upalakalin JN, Roberts MJ, et al. Blood markers for vasculogenesis increase with tumor progression in patients with breast carcinoma. Cancer Biol Ther 2003;2:255-6.

19 Pastorino U, Andreola S, Tagliabue E, et al. Immunocytochemical markers in stage I lung cancer: relevance to prognosis. J Clin Oncol 1997; 15:2858-65.

20 Ohta $Y$, Tomita $Y$, Oda M, et al. Tumor angiogenesis and recurrence in stage I non-small cell lung cancer. Ann Thorac Surg 1999;68:1034-8.

21 Fontanini G, Bigini D, Vignati S, et al. Microvessel count predicts metastatic disease and survival in non-small cell lung cancer. J Pathol 1995; 177:57-63.

22 Harpole DH Jr, Richards WG, Herndon JE 2nd, et al. Angiogenesis and molecular biologic substaging in patients with stage I non-small cell lung cancer. Ann Thorac Surg 1996;61:1470-6.

23 Macchiarini P, Fontanini G, Hardin MJ, et al. Relation of neovascularization to metastasis of non-small-cell lung cancer. Lancet 1992;340:145-6.

24 Ushiijima C, Tsukamoto S, Yamazaki K, et al. High vascularity in the peripheral region of non-small cell lung cancer tissue is associated with tumor progression. Lung Cancer 2001;34:233-41.

25 Kieser A, Weich HA, Brandner G, et al. Mutant $\mathrm{p} 53$ potentiates protein kinase $C$ induction of vascular endothelial growth factor expression. Oncogene 1994; 10:2207-12.

26 Dameron KM, Volpert OV, Tainsky MA, et al. Control of angiogenesis in fibroblasts by $\mathrm{p} 53$ regulation of thrombospondin-1. Science 1994;265: 1582-4.

27 Fontanini G, Boldrini L, Vignati $\mathrm{S}$, et $\mathrm{al}$. $\mathrm{Bcl} 2$ and $\mathrm{p} 53$ regulate vascular endothelial growth factor (VEGF)-mediated angiogenesis in non-small cell lung carcinoma. Eur J Cancer 1998;34:718-23.

28 Harpole DH Jr, Herndon JE, Wolfe WG, et al. A prognostic model of recurrence and death in stage I non-small cell lung cancer utilizing presentation, histopathology, and oncogene expression. Cancer Res 1995:55:51-6.

29 Brambilla E, Travis WD, Colby TV, et al. The new World Health Organization classification of lung tumors. Eur Respir J 2001;18:1059-68.

30 Hilbe W, Dirnhofer S, Oberwasserlechner F, et al. Immunohistochemical typing of non-small cell lung cancer on cryostat sections: correlation with clinical parameters and prognosis. J Clin Pathol 2003;56:736-41.

31 Asahara T, Murohara T, Sullivan A, ef al. Isolation of putative progenitor endothelial cells for angiogenesis. Science 1997;275:964-7.

32 Asahara T, Masuda H, Takahashi T, et al. Bone marrow origin of endothelial progenitor cells responsible for postnatal vasculogenesis in physiological and pathological neovascularization. Circ Res 1999;85:221-8.

33 Gunsilius E, Duba HC, Petzer AL, et al. Evidence from a leukaemia model for maintenance of vascular endothelium by bone-marrow-derived endothelial cells. Lancet 2000;355:1688-91.

34 Peichev M, Naiyer AJ, Pereira D, et al. Expression of VEGFR-2 and ACl 33 by circulating human CD34(+) cells identifies a population of functional endothelial precursors. Blood 2000;95:952-8.

35 DeLisser HM, Newman PJ, Albelda SM. Molecular and functional aspects of PECAM-1/CD31. Immunol Today 1994;15:490-5.

36 Pham I, Uchida T, Planes C, et al. Hypoxia upregulates VEGF expression in alveolar epithelial cells in vitro and in vivo. Am J Physiol Lung Cell Mol Physiol 2002;283:L1133-42. 